\title{
THE JEM-EUSO MISSION: OBSERVATION OF ULTRA-HIGH ENERGY COSMIC RAYS FROM SPACE
}

\author{
M. RICCI (for the JEM-EUSO COLLABORATION) \\ INFN, Laboratori Nazionali di Frascati, via E. Fermi, 40 \\ I-00044 Frascati (Roma), Italy \\ marco.ricci@lnf.infn.it
}

\begin{abstract}
The Extreme Universe Space Observatory on Japanese Experiment Module (JEMEUSO) is a science mission planned to be launched in 2017 to the International Space Station (ISS) to investigate the nature and origin of Ultra High Energy Cosmic Rays (UHECR) beyond energy $3 \times 10^{19} \mathrm{eV}$. JEM-EUSO is a wide-angle telescope (60 degrees full field of view) and consists of a high-transmittance Fresnel lenses $2.5 \mathrm{~m}$ in diameter, an advanced photo-sensitive detector at the focal surface and a suitable electronics. An infrared camera and a LIDAR system will also be used to monitor the Earth's atmosphere and provide significant information on cloud coverage. The present status of advancement of the mission is reported.
\end{abstract}

\section{Introduction}

JEM-EUSO will be the first long duration observatory in space devoted to the exploration of the Universe through the detection of Ultra High Energy Cosmic Rays (UHECR) and neutrinos with energy $\mathrm{E}>100 \mathrm{EeV} \cdot{ }^{1}$ It is intended to address basic problems of fundamental physics and high energy astrophysics by investigating the nature and origin of UHECR. The corresponding jump in statistics, due to the by-far larger exposure than presently ground-based running experiments, will clarify the origin (sources) of UHECR and, possibly, the particles mechanisms operating at energies well beyond those achievable by man-made accelerators. Furthemore, the spectrum of scientific goals of JEM-EUSO includes also as explorative objectives the detection of high energy gamma rays and neutrinos, the study of cosmic magnetic fields, and testing relativity and quantum gravity effects at extreme energies. In parallel, along the mission, JEM-EUSO will sistematically survey atmospheric phenomena over the Earth Surface.

In the JEM-EUSO concept (Fig. 1), the Earth's atmosphere is a giant detector. UHECRs collide with atmospheric nuclei and produce Extensive Air Showers (EAS). JEM-EUSO observes the fluorescence light emitted by the nitrogen molecules excited by the EAS charged particles and the reflected signal at ground of the Cherenkov emission associated with the shower development. Viewing from the ISS orbit, the $\pm 30^{\circ}$ Field-of-View (FoV) of the telescope corresponds to an observational area at ground larger than $1.9 \times 10^{5} \mathrm{~km}^{2}$. 


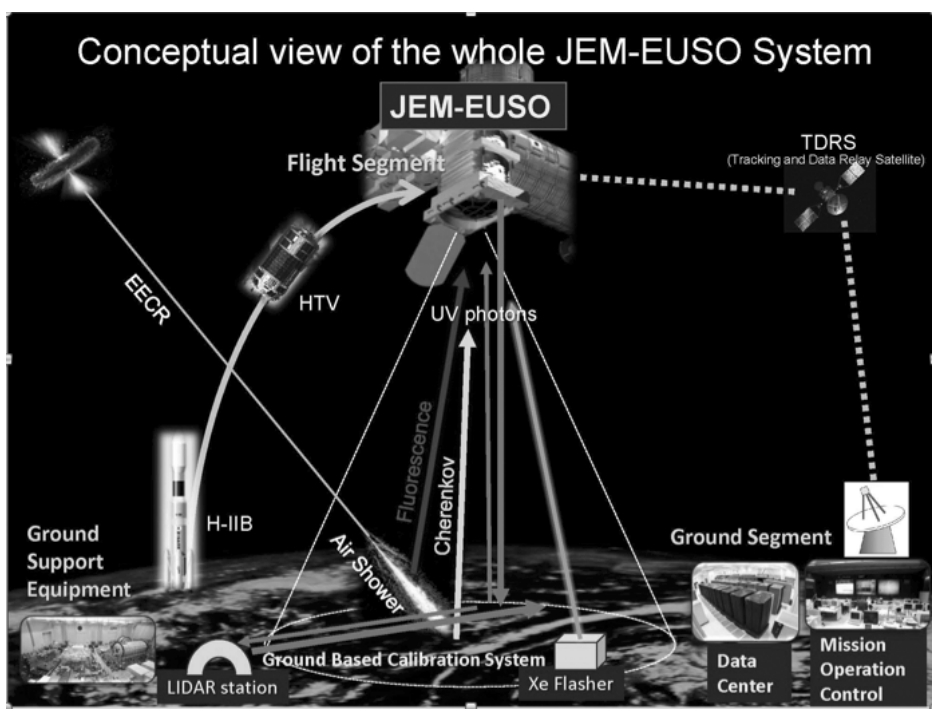

Fig. 1. Principle of the JEM-EUSO telescope to detect UHECRs.

The threshold energy of the detector is around $3 \times 10^{19} \mathrm{eV}$. Increase in exposure and energy threshold is realized by inclining the telescope from nadir to tilted mode, to extend the range of observation up to $10^{21} \mathrm{eV}$.

JEM-EUSO is foreseen to be launched in 2017 by a JAXA (Japanese Aerospace Exploration Agency) H2B rocket and conveyed by an H-II Transfer Vehicle on ISS and attached to the Exposure Facility of JEM.

The JEM-EUSO Collaboration is presently an international joint effort of 13 countries (Japan, USA, S.Korea, Mexico, Russia, Bulgaria, France, Germany, Italy, Poland, Slovakia, Spain, Switzerland), 77 Institutions and 270 researchers leaded by the Riken Institute, Tokyo.

\section{The Main Scientific Objectives}

The Cosmic Radiation can be considered as the Particle channel complementing the Electromagnetic one of conventional astronomy. Given current uncertainties, ${ }^{2,3}$ the expected number of events that will be detected by JEM-EUSO in a five year mission will range between 500 and 800 with energy above $5.5 \times 10^{19} \mathrm{eV}$. Such number of events makes possible the following targets: a) identification of sources by high-statistics arrival direction analysis; b) measurement of the energy spectra from individual sources to constrain acceleration or emission mechanisms. To compare with the largest presently running ground observatory, AUGER will collect, at the same energies, between 250 and 380 events in 15 years of operation; furthermore, as explained in Sect. 4, the annual exposure of JEM-EUSO is about 8-9 times larger than AUGER at $10^{20} \mathrm{eV}$. 
The photo-pion production of UHE proton interaction with the Cosmic Microwave Background (CMB) strongly suppresses the UHECR spectrum above $\sim 6 \times 10^{19} \mathrm{eV}$ (the GZK-cutoff ${ }^{4}$ ) effectively setting a horizon at nominally $\sim 100$ $\mathrm{Mpc}$, assuming a pure proton composition. The same applies for nuclei due to photo-disintegration. The result is that UHECRs sample the very local Universe, where the Large Scale Mass Distribution (LSMD) is inhomogeneous; therefore, the source distribution should emerge from the UHECR flux and arrival direction. Such analysis is being attempted by current operating ground observatories, however, the results are still uncertain.

JEM-EUSO has the great advantage of significantly increase the exposure keeping the declination dependence very low, thus contributing to considerably improve this kind of analysis.

If several sources are found with at least a dozen of observed events, the observed differences in spectral features among those sources, combined with a multi-wavelength approach, will provide direct clues on the identity of the sources and the acceleration mechanisms involved. In fact, the spectrum of a source located around $5 \mathrm{Mpc}$ should manifest a very low GZK cut-off effect, while a similar source around $50 \mathrm{Mpc}$ should show a much steeper energy spectrum. ${ }^{5}$

The pattern of the energy dependent distortions of the sources point spread functions as a result of the Galactic Magnetic Field, over the celestial sphere can be used to infer the large scale structure of the Galactic Magnetic Field itself.

\section{The JEM-EUSO Telescope}

The JEM-EUSO telescope ${ }^{6}$ is an extremely-fast, highly-pixelized, large-aperture and large-FoV digital camera, working in near-UV wavelength range (300-400 nm) with single photon counting capability. The telescope mainly consists of four parts: collecting optics, ${ }^{7}$ focal surface detector (FS), ${ }^{8}$ electronics ${ }^{9}$ and structure ${ }^{10}$ as shown in Fig. 2.

The optics is made by two curved double sided Fresnel lenses with 2.65 m external diameter, a precision middle lens and a pupil. The UV photons are focused onto the FS with an angular resolution of $\sim 0.07^{\circ}$. The FS detector (covered by $\sim 5000$ multi-anode photomultipliers) converts the incident photons to electric pulses, which are counted by the electronics in $2.5 \mu$ s Gate Time Units (GTU). When a signal pattern of an EAS is found, the trigger ${ }^{11}$ is issued and the intensity of the signal in the triggered and surrounding pixels is sent to the ground operation center. The list of the main parameters of JEM-EUSO telescope is reported in table 1.

The intensity of the fluorescence and Cherenkov light from EAS depends on the transparency of the atmosphere, the cloud coverage and the height of cloud top. For these reasons, JEM-EUSO will also account for an Atmospheric Monitoring (AM) system $^{12}$ to estimate as precisely as possible the sky conditions and the effective observing time with high accuracy. AM will consist of an infrared camera, a LIDAR system integrated by the slow data mode of the telescope itself. 


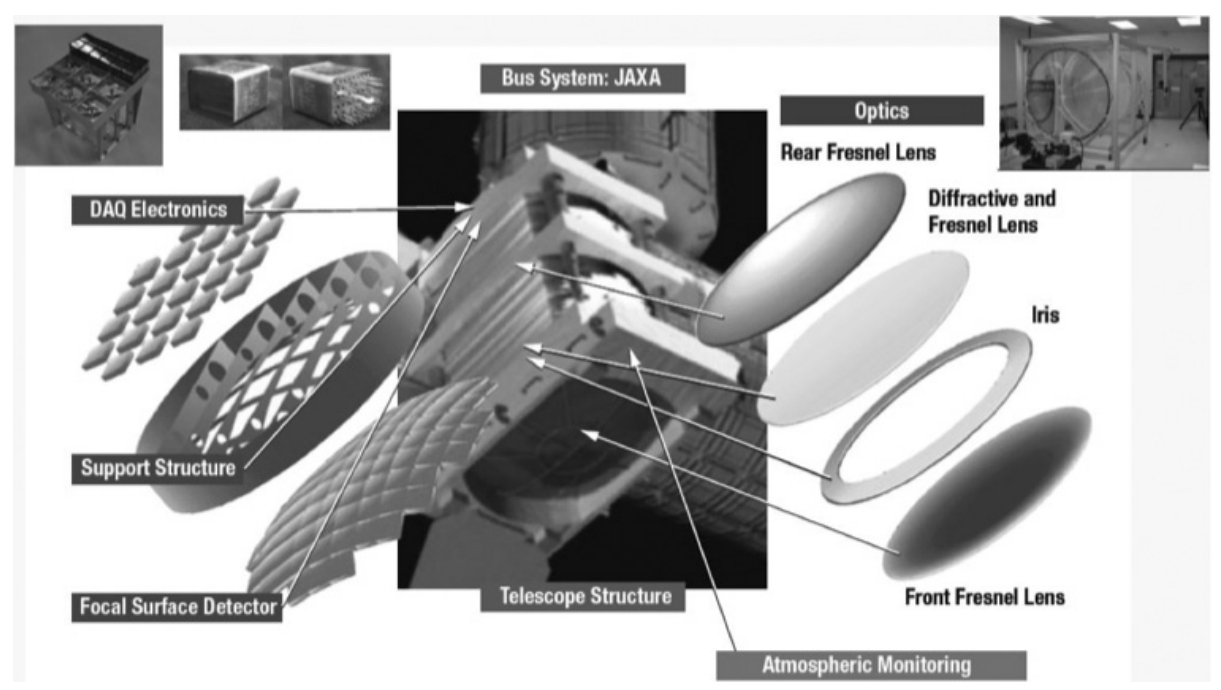

Fig. 2. Sketch of the main parts of the JEM-EUSO telescope.

Table 1. Parameters of JEM-EUSO telescope.

\begin{tabular}{cc}
\hline Field of View & $\pm 30^{\circ}$ \\
Observational area & $>1.9 \times 10^{5} \mathrm{~km}^{2}$ \\
Optical bandwith & $330 \div 400 \mathrm{~nm}$ \\
Focal Surface Area & $4.5 \mathrm{~m}^{2}$ \\
Number of pixels & $3.2 \times 10^{5}$ \\
Pixel size & $2.9 \mathrm{~mm}$ \\
Pixel size at ground & $\sim 550 \mathrm{~mm}$ \\
Spatial resolution & $0.07^{\circ}$ \\
Event time sampling & $2.5 \mu \mathrm{s}$ \\
Duty cycle $\times$ cloud impact & $\sim 14 \%$ \\
\hline
\end{tabular}

\section{The Observational Technique}

The main advantages of JEM-EUSO compared to any existing or planned groundbased experiment are the significant increase of aperture and the full-sky coverage with an almost uniform exposure. Moreover, as the EAS maximum develops for most zenith angles at altitudes higher than $3-5 \mathrm{~km}$ from ground, the measurements will be possible even in cloudy sky conditions. Compared to ground-based detectors, the duty cycle will be, therefore, mostly limited by the moon phase, while the cloud impact will be less important than for ground-based observations.

One of the key elements to estimate the performance of JEM-EUSO is the evaluation of its exposure. This can be factorized in three main contributions: the trigger aperture, the observational duty cycle and the cloud impact. The observational duty cycle, meant as the fraction of time in which EAS observation is not hampered by the brightness of the sky, has been evaluated by analyzing the measurements of the 


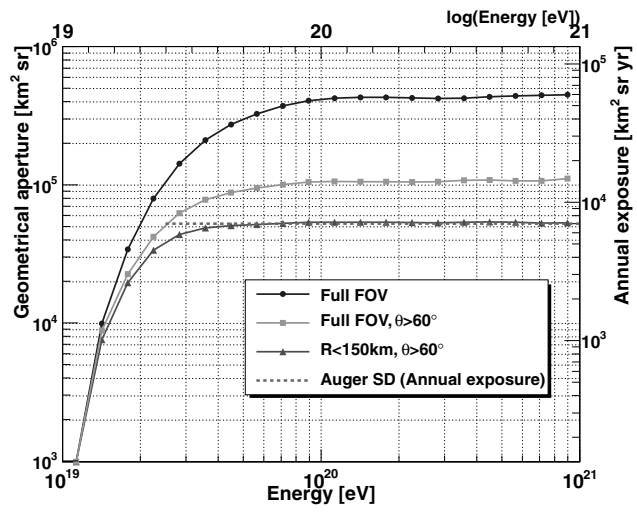

Fig. 3. Aperture and annual exposure of JEM-EUSO for different quality cuts.

Russian satellite Tatiana and rescaling them to the ISS orbit ${ }^{13}$ and it accounts for $\sim 20 \%$.

The peculiarity of the observation from space is the possibility of observing CRs also in some cloudy conditions (i.e. if the shower maximum is above the cloud top), which is tipically not the case for ground-based telescopes. Studies and simulations on cloud distributions and evaluation of cloud impact have been carried out, ${ }^{1415}$ The result indicates that the average fraction of the observational time where the measurement will not be hampered by atmospheric factors is $\sim 70 \%$. This number, convoluted with the $20 \%$ duty cycle observational time, provides a final $14 \%$ multiplication factor to be applied to the aperture to determine the exposure.

Figure 3 shows the full aperture, and annual exposure of JEM-EUSO in nadir mode for the full FoV of the detector together with different quality cuts: ${ }^{16} 80-90 \%$ aperture is already reached at energies $\sim 2-3 \times 10^{19} \mathrm{eV}$ when the footprint of the shower is located in the central part of the $\mathrm{FoV}(\mathrm{R}<125 \mathrm{~km}$ from nadir $)$ and with zenith angles $\theta>60^{\circ}$, and it slightly increases at $\sim 5 \times 10^{19} \mathrm{eV}$ if the entire FoV is considered.

In the most stringent conditions, JEM-EUSO has an annual exposure equivalent to Auger $\left(\sim 7000 \mathrm{~km}^{2} \mathrm{sr}\right.$ yr) while it reaches $\sim 60000 \mathrm{~km}^{2}$ sr yr at $10^{20} \mathrm{eV}, 9$ times Auger equivalent.

\section{Planned Work}

Besides simulations and performance studies, several technological prototypes of the optical system, of the mechanical support structures and of the front-end electronics and data acquisition have been developed and are in progress. In the forthcoming two years a couple of tests on a scaled prototype of the telescope made by a system of Fresnel lenses and one single PDM (Photo Detector Module) unit fully equipped are being planned. The first one will be performed at the Telescope Array site in Utah where such a prototype will be deployed for calibration purposes. Then, a similar 
one will fly on stratospheric balloons (managed by CNES, France) for engineering purposes such as the verification of the trigger electronics and the measurement of the nightglow background in conditions as close as possible to what is expected from ISS, and possibly to observe the first EAS from space.

\section{References}

1. Y. Takahashi, New Journal of Physics 11, 065009 (2009); T. Ebisuzaki et al., Nucl. Phys. B (Proc. Suppl.) 175-176, 237 (2008).

2. J. Abraham et al., Science 318(5852), 938 (2007); J. Abraham et al., Astrop. Phys. 29(3), 188 (2008); P. Abreu et al., Astrop. Phys. 34(5), 314 (2010).

3. R.U. Abbasi et al., Astrop. Phys. 30(4), 175 (2008).

4. K. Greisen, Phys. Lett. 16, 148 (1966); G.T. Zatsepin \& V.A. Kuz'min JETP Phys. Lett. 4, 78 (1966).

5. G. Medina Tanco et al., Proc. 32 ${ }^{\text {nd }}$ ICRC, Beijing \#0956, (2011).

6. F. Kajino et al., NIM A 623422 (2010).

7. A. Zuccaro Marchi et al., Proc. 32 ${ }^{\text {nd }}$ ICRC, Beijing \#0852, (2011).

8. Y. Kawasaki et al., Proc. 32 $2^{\text {nd }}$ ICRC, Beijing \#0472, (2011).

9. M. Casolino et al., Proc. 32 ${ }^{\text {nd }}$ ICRC, Beijing \#1219, (2011).

10. M. Ricci et al., Proc. 32 ${ }^{\text {nd }}$ ICRC, Beijing \#0335, (2011).

11. O. Catalano et al., Proc. $31^{\text {nd }}$ ICRC, Lodz \#0326, (2009).

12. A. Neronov et al., Proc. 32 ${ }^{\text {nd }}$ ICRC, Beijing \#0301, (2011).

13. P. Bobik et al., Proc. 32 ${ }^{\text {nd }}$ ICRC, Beijing \#0886, (2011).

14. F. Garino et al., Proc. 32 $2^{\text {nd }}$ ICRC, Beijing \#0398, (2011).

15. L. Saez Cano et al., Proc. 32 $2^{\text {nd }}$ ICRC, Beijing \#1034, (2011).

16. K. Shinozaki et al., Proc. 32 $2^{\text {nd }}$ ICRC, Beijing \#0979, (2011). 